\title{
Escuela y comunidad: tendencias, incertidumbres y elementos para una discusión pendiente ${ }^{1}$
}

\author{
Rodrigo Velásquez B. ${ }^{*}$, Christian Miranda J. **
}

\begin{abstract}
RESUMEN
En este artículo se presentan los resultados de una revisión bibliográfica que busca exponer los hallazgos de investigaciones sobre el vínculo entre escuela y comunidad, identificando en los investigadores dos focos de interés relacionados con el tema: a) ciudadanía y participación y b) la familia en la escuela. A partir de estas tendencias, se constata que las escuelas, guiadas por una racionalidad instrumental, restringen la participación y la toma de decisiones a estudiantes y familias, dificultando la construcción de una verdadera comunidad y que los profesores son percibidos como actores clave para propiciar o restringir la participación.
\end{abstract}

Palabras clave: Escuela, comunidad, participación, familia.

\section{School and Community: Trends, uncertainties and elements for a pending discussion}

\begin{abstract}
This article presents results from a bibliographical review which intends to show findings of a research about links between school and community, identifying two perspectives of interest related to the subject in the researchers: a) citizenship and participation, and b) family in school. From these trends, it is validated that schools guided by an instrumental rationality limit the involvement and decision-making actions of students and families, making the construction of a true community more difficult and perceiving teachers as key figures who contribute or limit participation.
\end{abstract}

Keywords: School, community, participation, family.

I Artículo que forma parte de un estudio mayor denominado "Hacia un modelo se seguimiento de la formación permanente de profesores", financiado por la CONICYT a través del Programa FONDECYT ( ${ }^{\circ}$ II 40827$)$.

* Profesor de Filosofía, Mg. en Educación, U. de Chile. E-mail: rodrigovelasquezb@gmail.com

Doctor en Ciencias de la Educación. Profesor Asociado de la Facultad de Ciencias Sociales, Universidad de Chile. E-mail: christian.miranda@u.uchile.cl 


\section{Introducción}

Hablar de comunidad escolar parece ser hoy un lugar común en el mundo de la educación. El concepto se ha instalado en el lenguaje de quienes participan e investigan la escuela y se utiliza casi como un sinónimo de ella, ya que, a diferencia de otras instituciones, su finalidad está íntimamente ligada a su condición de comunidad. Si miramos los sentidos que se le atribuyen a la escuela en la modernidad, desde la preparación para el mundo laboral hasta la formación de ciudadanos capaces de participar en una sociedad paulatinamente más democrática, podemos suponer que la educación de los estudiantes es algo más que simple instrucción y que las relaciones y experiencias que se dan al interior de los centros educativos deben apuntar hacia el desarrollo y consolidación de un cierto modelo de ser humano y de sociedad, por lo que es preciso comprenderlas como organizaciones que "están basadas en relaciones e interacciones con significaciones morales y no sólo contractuales, en que los miembros se sienten pertenecientes a un grupo determinado, con relaciones afectivas fuertes y que comparten valores" (Sergiovanni, 1994, en Ansaldo San Martín, 2015, p. 68). Sin embargo, resulta menester detenerse a reflexionar y problematizar el concepto de comunidad, pues tiende a darse por hecho que las escuelas son comunidades antes de examinar si efectivamente lo son. Esto cobra relevancia en medio del actual sistema educacional chileno, objeto de cuestionamientos y exigencias por parte de ciertos sectores que, desde la década de I990 en adelante, ha puesto en marcha políticas educativas como la Jornada Escolar Completa, que "recomendaba la conformación de centros de padres y de estudiantes como forma de participación de los miembros de la comunidad escolar" (Ansaldo San Martín, 2015, p. 68), o proyectos recientes como la Política Nacional de Convivencia Escolar (Mineduc, 2015), solo por nombrar algunos, con el fin de abrir, democratizar y consolidar espacios de participación al interior de las comunidades escolares.

Para ello, se desarrolla una breve revisión conceptual del término "comunidad" enfocada en el ámbito educativo, con el objetivo de comprender qué significa que las escuelas sean comunidades. En segundo lugar se expone la importancia del tema en el contexto 
educacional chileno. Luego, se presentan los resultados del análisis de las investigaciones chilenas acerca del problema, en las que aparecen dos tendencias o focos principales para referirse al tema: a) la comunidad ligada a la ciudadanía y participación y b) la comunidad vista desde la relación escuela-familia. Por último, intentamos responder qué elementos propios de una comunidad poseen las escuelas para permitirnos hablar de comunidad educativa, a la vez que nos referimos a algunos vacíos epistemológicos en el conocimiento producido en esta área, los que, a nuestro juicio, interpelan a la formación y el ejercicio docente.

\section{Antecedentes}

El concepto más tradicional de comunidad se refiere a relaciones territoriales situadas y delimitadas que fundan un vínculo de proximidad entre diversos sujetos, generando sentimientos de afecto e identificación: implica que los individuos se conozcan, generen intereses compartidos y sus problemas sean resueltos en común (Krichesky, 2006). Sin embargo, esta mirada tradicional hoy aparece restringida, en un mundo globalizado que reconoce una multiplicidad de comunidades al interior de un mismo espacio geográfico, por lo que la conformación de comunidades se vuelve un proceso complejo, pues lo sustancial de ellas es la constitución e identificación con una red de significados comunes, que puede ir desde la base territorial hasta fines e intereses compartidos (Subirats, 2003; Neirotti y Poggi, 2004; Krichesky, 2006). A partir de este nuevo escenario se ha impulsado una ampliación del concepto, tal como es descrito por Vargas Cetina (2002, Cit. en Krichesky, 2006) al referirse a comunidades basadas en la diferencia, para distinguirlas de aquellas propias de la modernidad. Así pues, actualmente las comunidades construidas en la diferencia, es decir, en contextos plurales y multiculturales, se definen como espacios habitados, concreta o virtualmente, por sujetos diversos que desarrollan proyectos y construyen fines en común -sin llegar necesariamente a la homogeneización total de sus integrantes-, que logran un mutuo reconocimiento al establecer ciertas creencias, valores, conocimientos o metas comunes y que generan un sentido de identidad con lo común. 
Desde esta perspectiva, es importante destacar la diferencia con el concepto de "sociedad", que sería más bien un grupo de personas, que comparten un espacio territorial, pero que no constituyen redes de proximidad ni identificación que los vinculen simbólicamente, estableciendo relaciones utilitarias entre sus integrantes, característica propia de sociedades conducidas por una racionalidad instrumental (Subirats, 2003).

La premisa tras esta concepción remozada de comunidad es la participación entendida como decisión: para construir estos intereses comunes es necesario poder participar activamente y tener la posibilidad de tomar decisiones. De esta manera, la comunidad implica compartir un espacio, físico o virtual, construido intersubjetivamente, cuyo fin es producir una identificación con los otros y mantener un sentido de mismidad (Krichesky, 2006).

Desde esta concepción de las comunidades, en el ámbito educativo se han desarrollado conceptos como "comunidades de aprendizaje", "comunidad educativa”, "escuela abierta”, "escuela inclusiva”, etc., que intentan dar cuenta de este fenómeno y desarrollar proyectos de intervención que permitan abrir y democratizar el espacio de la escuela para la formación ciudadana, mejorar los aprendizajes y brindar apoyo a estudiantes y familias que viven en condiciones de vulnerabilidad social (Rivera y Milicic, 2006), sectores en los que urgiría pasar de una escuela cerrada -volcada sobre sí misma- a una abierta a la comunidad, que reconoce el valor educativo de otros agentes sociales y que se asume, ella misma, como un espacio comunitario, permitiendo que sus integrantes participen activamente en el diseño y la construcción de lo común. Se torna imprescindible, pues, abrir la escuela y comprenderla como una comunidad no excluyente que integre a otros actores sociales como agentes educativos, lo que se denomina "escuelas abiertas": "la tendencia de las escuelas abiertas implica -entre otros aspectos- una apertura al medio, para lo cual la institución debe ser sensible a las demandas y expectativas del entorno, y regular su acción, en muchos casos, en una negociación permanente en la que redefine y explicita los términos del intercambio" (Krichesky, 2006, p. I6), esto es, redefinir el concepto de comunidad educativa. 
Así entendida, asumimos que la comunidad escolar es aquella institución que, desde una gestión democrática, participativa e inclusiva, logra desarrollar proyectos y perseguir fines comunes generando identificación entre sus miembros, sin por ello imponer intereses exógenos, sino que, por el contrario, dichos fines serían el resultado del diálogo y la participación.

La relación escuela-comunidad es, en Chile, a nuestro juicio, uno de los pilares en los que se sostiene y uno de los horizontes hacia el que apunta el actual discurso educativo, en tanto se habla habitualmente de "comunidad educativa" o "comunidad escolar" como sinónimos de lo que las escuelas son por antonomasia o de lo que deberían llegar a ser. La importancia de esta díada queda de manifiesto desde ya en el mismo lenguaje, puesto que referirse a las escuelas como comunidades implica reconocer ciertas características propias, inherentes a su naturaleza; no se trata de decir que una escuela, por ejemplo, es grande, vulnerable o lejana, asignándole un adjetivo; antes bien, al hablar de "comunidad educativa", la categoría "comunidad" es parte de una construcción conceptual que toma la forma de un sustantivo, y, por ende, no debería ser algo accidental ni transitorio, sino parte constitutiva de la institución escolar.

Pero más allá de la interpretación estrictamente conceptual y tal como adelantamos en la discusión del término, si las escuelas se comprenden a sí mismas como comunidades, si sus integrantes tienen experiencias participativas y deliberativas para la construcción de significados y fines de la institución, entonces brindan oportunidades de aprendizaje para la convivencia en una sociedad democrática y para la formación de ciudadanos activos y responsables, uno de los objetivos declarados en el sistema educacional chileno.

Comunidad y democracia aparecen imbricadas mutuamente (Díaz Barrera, 2010), pues "una sociedad democrática vigorosa, necesita desarrollar en sus miembros la idea que se trata de un proyecto común $[\ldots]$. Ese tipo de compromiso cívico sobre el que se soporta la democracia requiere que los miembros de la sociedad se sientan vinculados con las demás personas que forman parte de su comunidad” (Redón: 20I I, p. 459). Y la escuela adquiere un 
papel fundamental en esto, ya que es ahí donde pueden desarrollar experiencias significativas para la vida democrática. Las escuelas tienen, entonces, la labor de preparar ciudadanos que se integren a la sociedad y la enriquezcan, trabajando mancomunadamente para cumplir esta meta y es al interior de ellas que este proceso debe ser llevado a la práctica para que se traduzca en aprendizajes significativos. Díaz Barrera (2010) defiende la necesidad de desarrollar desde temprana edad este tipo de aprendizaje en los niños y niñas, quienes debiesen ser educados en y por la democracia, de modo que sean capaces de generar mecanismos de inclusión por sí mismos. Entre las iniciativas para tales efectos, Muñoz (20II) destaca el Consejo Escolar como una oportunidad de aprendizaje ciudadano mediante la experiencia; lo que comparten Martínez et al. (2010) en una revisión de estudios previos sobre la ciudadanía en los jóvenes, al afirmar que la formación de actitudes y habilidades ciudadanas debe desarrollarse desde la experiencia y la cotidianidad de niños y jóvenes, pues la escuela es un lugar propicio para ello. Por lo tanto, quienes habitan y conviven en la comunidad escolar -estudiantes, profesores, equipos directivos y familias, entre otros- han de ser convocados a participar y dialogar para cumplir con el fin último del acto educativo.

No obstante, en la actualidad el despliegue efectivo de este discurso enfrenta cuestionamientos, en primer lugar, porque el contexto en el que nacieron las escuelas ha sufrido cambios, ya que la modernidad que las vio nacer hoy es objeto de críticas o, al menos, de cierto escepticismo: "la afirmación de que el progreso es la marcha hacia la abundancia, la libertad y la felicidad, y que estos tres objetivos están fuertemente ligados entre sí no es más que una ideología constantemente desmentida por la historia" (Touraine, 2012, p. I0). En términos sociológicos hoy asistiríamos a lo que Bauman (2012) denomina "modernidad líquida", es decir, la arremetida de la modernidad contra sí misma, con un objetivo que viene inscrito en su propia naturaleza: "derretir los sólidos". Algunas de las características de esta nueva "fase" de la modernidad son despojar a las acciones humanas -a partir de la predominancia de la economía- de su dimensión ética y terminar paulatinamente con la confianza y la seguridad proporcionada por el conocimiento, las 
relaciones sociales, los vínculos laborales, etc. Se trata de una época en la que los sólidos -los grandes relatos, las grandes industrias, las clases sociales claramente definidas, etc.- comienzan a desaparecer y son reemplazados por un mundo líquido, fugaz y de relaciones inestables e individuales. El sujeto se aleja de las formas tradicionales de vida - por ejemplo, la comunidad-y comienza un recorrido que lo empuja a caminar solo, liberándose de las antiguas ataduras sociales que lo liaban a otros para habitar el mundo: "la concepción occidental más vigorosa de la modernidad, la que tuvo efectos más profundos, afirmaba que la racionalización imponía la destrucción de los vínculos sociales, de los sentimientos, de las costumbres, y de las creencias llamadas tradicionales" (Touraine, 2012, p. I8), lo que pondría en tela de juicio la efectividad de la labor asignada a la escuela y de los principios y fines de la educación que aparecen en la Ley General de Educación, particularmente aquella que busca la formación de una ciudadanía activa y democrática, la que supone, como hemos dicho, que la escuela es o debe ser una comunidad.

Los cambios en la modernidad y las críticas que esta ha recibido, en tanto discurso y proyecto filosófico-político, han traído consecuencias para la institución escolar, la que ha sido objeto de cuestionamientos e interpelada para hacerse cargo de la dimensión no disciplinaria de la educación, aquella vinculada a la formación de personas que se involucren en la vida comunitaria y social en un contexto democrático. En Chile se habla de falta de participación, prácticas poco democráticas, segregación y problemas de convivencia en las escuelas, ejemplos del complejo panorama que los centros educativos se deben enfrentar. Ante esto, se han implementado y se siguen diseñando políticas públicas capaces de responder a estos requerimientos, que tratan de hacerse cargo de los fines de la educación consagrados en la Ley General de Educación. Creemos que todo esto exige fijar la mirada en un concepto que sirve de soporte para propiciar la participación, el diálogo y la convivencia democrática en vistas del bien común: "la comunidad”. El desafío actual en sociedades que han dejado atrás la etapa sólida de la modernidad, sociedades multiculturales con escuelas compuestas por un estudiantado diverso y heterogéneo, es construir una escuela capaz de constituirse en una comunidad, pues de ese modo permitiría el desarrollo efectivo de una formación más 
amplia, demandada por diversos actores y enarbolada en los discursos educativos. Se requiere, por tanto, avanzar hacia la transformación de las escuelas en verdaderas comunidades educativas, con todo lo que ello implica. Para aportar en esta tarea se propone examinar qué nos dicen las investigaciones chilenas a partir de la búsqueda y análisis de estudios sobre el vínculo escuela-comunidad, con el fin de identificar cuáles son los focos o tendencias que han predominado en estos estudios, develar posibles vacíos epistemológicos y aportar elementos para la discusión en torno a este problema. Se tratará de abordar las siguientes preguntas con base en lo que se entiende aquí por "comunidad": ¿cuáles son las principales tendencias que se identifican en las investigaciones chilenas? ¿Qué características de las comunidades presentan las escuelas en Chile? Las escuelas chilenas, ¿son comunidades?

\section{Metodología}

El presente trabajo corresponde a una revisión bibliográfica, un tipo de estudio que, siguiendo a Roussos (20II), tiene como objetivo principal analizar y sintetizar la información sobre un tema seleccionado, y, a partir de ahí, evaluar distintos aspectos del mismo, tal como el grado de conocimiento, síntesis de diferentes posturas, aspectos comunes que no han sido comparados, etc. Toda revisión bibliográfica debe establecer criterios de recopilación del material, para establecer límites y hacer que el trabajo sea abordable en medio de la gran cantidad de información disponible. En este caso, los criterios de recopilación fueron tres: temático, cronológico y científico. Se asume pues que en este tipo de trabajos la recopilación y el rastreo bibliográfico no puede ser completamente exhaustivo, ya que existen diferentes variables que restringen la búsqueda y el acceso a la información, de modo que no se pretende agotar el problema; por el contrario, la intención es abrir el debate en esta área a partir de algunas investigaciones seleccionadas, asumiendo que deben existir otras, igual de valiosas, que no pudieron ser incorporadas.

En este sentido, nos propusimos buscar investigaciones chilenas que aborden como tema central el vínculo escuela-comunidad y, a partir de su análisis, identificar las tendencias que presenta la literatura nacional 
al respecto para la discusión del conocimiento aportado por estos estudios. Tal como aparece más atrás, este tema ha ido volviéndose más relevante en Chile, por lo que solo se buscó investigaciones nacionales, pues antes de aventurar un estudio comparado es preciso rastrear y revisar la producción científica local y levantar una suerte de diagnóstico respecto del tema. Además, se excluyó investigaciones sobre comunidades de aprendizaje u otras ligadas a la educación en comunidades indígenas, por ejemplo. Por otro lado, cuando se habla del criterio cronológico nos referimos a que se acotó la amplitud de publicación de los trabajos revisados a los últimos I0 años, es decir, solo consideramos investigaciones publicadas desde 2006 en adelante para contar con información actualizada del tema. Por último, para encontrar los trabajos se utilizaron las bases de datos EBSCO, SciELO, SCOPUS y Schoolar Google, con el fin de seleccionar aquellos que hayan pasado por la revisión de un comité editorial y estén publicados en revistas indexadas. Inicialmente se buscó tesis de pre y posgrado en el Catálogo Bello de la Universidad Chile; sin embargo, no se identificó ningún trabajo relevante para nuestros fines, de modo que se optó por no seguir indagando en tesis y concentrar la búsqueda en artículos publicados por revistas. A través de los motores de búsqueda de cada página web se introdujeron, en primera instancia, las palabras clave: "comunidad", "escuela y comunidad", "comunidad(es) de aprendizaje”, "comunidad educativa”, a las que se sumaron "participación”, "ciudadanía”, "escuela inclusiva” y "familia y escuela", pues a medida que se avanza se van ampliando o modificando las palabras clave.

Se encontró un total de 23 artículos vinculados con nuestro problema, de los cuales solo II cumplían con los tres requisitos para ser incluidos, es decir, tenían como tema central la díada escuela-comunidad, fueron publicado en los últimos IO años en revistas que aseguran su calidad. De los II escritos seleccionados, 8 corresponden a investigaciones cualitativas (Ansaldo San Martin, 20I5; Cárcamo, 2008; Flanagan et al., 20I0; Muñoz, 20I I; Prieto, 2005; Redón, 2010, 20I I; Rivera y Milicic), 2 a sistematizaciones y análisis de estudios previos (Ferrada y Flecha, 2008; Martínez, 2010) y un ensayo a partir del análisis conceptual y de políticas públicas (Gubbins, 2012). 
La metodología que se utilizó para analizar la información recopilada fue la Teoría Fundamentada (Strauss y Corbin, 2002), pues al encontrar pocas investigaciones que se enfoquen directamente en el tema - de hecho, la más precisa en términos temáticas es la de Ansaldo San Martín (2015), que se define justamente como un trabajo exploratorio ante los escasos estudios al respecto-, esta metodología permite proceder inductivamente para la proposición de una teoría, con el fin de comprender un fenómeno partiendo de los datos empíricos, lo que se justifica en tanto no nos encontramos con ninguna teoría sobre el tema.

Una vez seleccionados los textos, se hizo un microanálisis en el que se destacaron los temas y conceptos que aparecían. Paralelamente, estos temas y conceptos se fueron agrupando por cercanías temáticas y se obtuvieron diversos códigos o categorías emergentes que contenían y sintetizaban la información procedente del microanálisis, lo que se denomina categorización abierta: concepciones sobre la ciudadanía, posibilidades y obstáculos para la participación, familia, expectativas sobre los roles al interior de la escuela y aprendizaje fueron las categorías emergentes. A continuación, estas categorías emergentes se redujeron a dos grandes categorías de análisis, "ciudadanía y participación" y "la familia en la escuela", lo que corresponde a la categorización axial, mediante el ajuste y clasificación en categorías y subcategorías que permitían comprender el fenómeno de manera más clara.

Por la dificultad de encontrar investigaciones que fuesen más precisas sobre el tema de esta revisión bibliográfica no se desarrolló la categorización selectiva, esto es, la construcción de un orden y jerarquía que permitiera elaborar una teoría sobre el tema de este artículo. Sin embargo, la categorización axial permitió identificar las tendencias que muestran las investigaciones aquí revisadas, es decir, los focos de interés que tienen los investigadores y qué nos dicen sobre ellos.

\section{Resultados}

Pese a la centralidad que ha adquirido este tema, en la búsqueda aquí realizada no se encontró una línea científica consolidada, al menos 
no en las bases de datos utilizadas, que se dirija específicamente a la temática escuela-comunidad, lo que se puede constatar, en primer lugar, a través de los temas y objetivos centrales de estas investigaciones (participación, ciudadanía, familia, prácticas escolares, aprendizaje) referidos, en general, indirectamente, a nuestra problemática, y, en segundo lugar, con las reducidas publicaciones chilenas en revistas indexadas. No obstante estas dificultades y la acotada publicación de investigaciones en Chile, se logró identificar al menos dos tendencias que se refieren a elementos de la relación escuela-comunidad, las que se clasificaron en dos grandes categorías: a) ciudadanía y participación, y b) la familia en la escuela. Nos referiremos a cada una de ellas intentando mostrar sus características generales.

\section{Ciudadanía y participación}

La primera tendencia que aparece en esta revisión bibliográfica está constituida por aquellas investigaciones que tienen como foco de interés indagar en torno a la participación y la ciudadanía, dos conceptos relacionados con el de "comunidad", en tanto esta presupone una participación activa por parte de sus miembros para la construcción de los significados.

De esta manera, se han desarrollado trabajos que, en el marco de la escuela como comunidad, han indagado y profundizado principalmente acerca de las percepciones y valores otorgados a la ciudadanía como objetivo de la formación escolar y la participación como mecanismo para fomentar la ciudadanía, por parte de profesores, estudiantes y apoderados como integrantes de la supuesta comunidad escolar.

Las investigaciones dan cuenta de una aceptación de la ciudadanía como misión de la educación y la escuela como espacio para su formación, mediante instancias como los Consejos Escolares, Centros de Estudiantes, por ejemplo. Sin embargo, conviven distintas concepciones sobre qué significa ser ciudadano, junto con una percepción de una participación más bien limitada por parte de estudiantes y apoderados. A partir de esto último, se identifican algunos factores que incidirían en la instalación limitada y simbólica. 
En general, las concepciones que profesores y estudiantes poseen acerca de la participación nos hablan, por una parte, del reconocimiento del nexo entre ciudadanía y escuela, y por otra de una valoración positiva de esta relación, pues permitiría desarrollar las aptitudes ciudadanas y democráticas presentes en los discursos educativos. En el caso de los profesores (docentes en formación y académicos universitarios que los forman) en la Universidad del Bío-Bío "afirman que existe un estrecho vínculo entre las prácticas pedagógicas y el desarrollo de la ciudadanía” y valoran la "promoción de la ciudadanía como un elemento gravitante y esencial de la formación de profesores” (Cárcamo, 2008, p. 42). En la escuela esta afirmación se mantiene por parte de docentes y estudiantes, y "en este sentido, la democracia se reconoce como la posibilidad de sentirse convocado por el bien común, ya que las organizaciones sociales nacen de sus propias necesidades, validadas por la comunidad escolar" (Muñoz, 20I I, p. 46), algo que hipotéticamente debiese reflejarse, por ejemplo, en el Consejo Escolar, espacio que, pese a sus limitaciones, tiene el potencial para convertirse en un instancia para la participación y la democratización, atendiendo así las exigencias del estudiantado (Muñoz, 20II); en el Centro de Alumnos, que se muestra como una organización estudiantil vinculada al ejercicio de la ciudadanía, o en el Consejo de Curso, lugar en donde los estudiantes pueden expresar sus opiniones y tomar decisiones (Flanagan et al., 2010). Por otro lado, los profesores y las familias de algunos establecimientos también sueñan con una escuela más participativa y abierta a la comunidad, una escuela en que la vida al interior de ella no solo se limite a lo estrictamente académico:

La escuela que conciben como una comunidad, es una escuela abierta a las familias de alumnos y profesores, con miras a satisfacer necesidades de recreación en horarios más allá de lo obligatorio y que por lo tanto, propicie la participación de todos en igualdad de condiciones y bajo el propósito de descansar y divertirse, como también de capacitarse mejor para atender las necesidades de los hijos y las propias (Rivera y Milicic, 2006, p. I27)

Ahora bien, a pesar de estas visiones que aprueban la participación como aspecto importante a desarrollar en el contexto de una 
educación que busca la formación de ciudadanos que se interesen por el bien común y así formar una comunidad, los significados atribuidos manifiestan cierta limitación. Por ejemplo, en la investigación de Flanagan et al. (2010) los profesores caracterizan a un "ciudadano" como aquel que está informado de lo que ocurre en la sociedad y participa en procesos eleccionarios (políticos y escolares) mediante el voto, mientras que los estudiantes lo definen en función de los contenidos que debe poseer como "el cuidado de la ciudad, conocer y obedecer las normas y leyes y saber procedimientos característicos de un país como lo es el sufragio" (Flanagan et al., 2010, p. 127). En este caso, tanto para estudiantes como para docentes la membrecía otorgada por la ciudadanía se obtiene formalmente habiendo nacido en un país, teniendo I8 años y estando inscrito en los registros electorales, y si bien los profesores conciben el formar ciudadanos con informar para participar, los estudiantes la definen "como la enseñanza o instrucción respecto a normas y leyes" (Flanagan et al., 2010, p. I29), con el objetivo de buscar el bien para el país. "Así, para ambos actores escolares, la ciudadanía se vincularía con una entrega y protección de derechos al individuo, y correspondería con lo que Dueñas (200I) entiende como ciudadanía política e individual, constituida por contenidos formales relacionados con la Educación Cívica, y en donde la "participación se operacionaliza en el acto de sufragio” (Flanagan et al., 2010, p. I33). Estas concepciones parecen estar en sintonía con lo expuesto por Cárcamo (2008) quien, a pesar de afirmar que hay al menos dos perspectivas de ciudadanía en juego en la formación de profesores, reconoce que impera la visión de la ciudadanía política.

Las prácticas escolares que buscan generar mayor participación serían restringidas, pues, en el caso del Consejo Escolar, "se valida una participación simbólica al decidir sobre pequeñas cosas que no tienen incidencia en los aspectos esenciales de la escuela” (Muñoz, 2012, p. 46). Como vemos, la imposibilidad de tomar decisiones importantes implica mirar críticamente el discurso de la participación. Siguiendo a Muñoz (20II), se distinguirían dos construcciones distintas respecto de la participación: las macropolíticas provenientes del Ministerio de Educación, las políticas públicas y las micropolíticas resultantes de la interacción de los actores al interior de la escuela. 
Estas últimas buscan democratizar los procesos escolares y propiciar una participación auténtica, mientras que las políticas de participación provenientes del Ministerio no serían más que mecanismos de control que permiten mantener la gobernabilidad, lo que se expresaría en las distintas instancias de participación escolar.

Por su parte, Prieto (2005) profundiza la percepción de estudiantes de secundaria, revelando que estos conciben los espacios de participación como restringidos, consecuencia de una formación directiva y rutinaria, sin que sea posible desarrollar la reflexión, es decir, la participación es limitada por una concepción y una práctica pedagógica reproductivas de contenidos que no potencian la reflexión, pues se pide repetir información, lo que obliga a los estudiantes a adaptarse a los requerimientos de los profesores, quienes buscarían ganar la obediencia del alumnado. En esta misma línea, se constata en otro estudio que la participación en los centros educativos es prácticamente nula, negando, en casi todos los casos, los espacios de diálogo y participación: "los estudiantes no deciden casi nada, ni el turno de habla, lugares de asiento, contenidos, actividades, espacio, tiempo escolar" (Redón, 20II, p. 462). Ante este escenario, se afirma que la escuela erróneamente es llamada "común-unidad", pues las prácticas que se desarrollan en su interior atentan contra el diálogo, la participación, la formación ciudadana y se enfila más bien hacia el control y el disciplinamiento, minando la construcción de significados por parte de los estudiantes, ya que el control normativo atenta contra el sentido de pertenencia de los grupos estudiados, lo que trae como consecuencia la dificultad de desarrollar valores ciudadanos, pues estos son fruto de un ejercicio social y no de una labor individual. Esta ausencia de participación, discusión y construcción de horizontes compartidos es observada también por Ansaldo San Martín (2015, p. 80):

Los valores y visones de mundo son poco compartidas entre miembros de lo que se llama la comunidad escolar, no en el sentido de que sean opiniones distintas, sino en que la escuela no es un lugar de puesta en común de esos valores. Hay pocas instancias y encuentros para desplegar, dar a conocer y discutir consensos y disensos, lo que va minando las posibilidades de una comunidad con enfoque ético. 
¿Por qué el discurso de la participación, la ciudadanía y la democracia, pilares para la construcción de comunidad, no se despliega satisfactoriamente? Sin intentar construir una teoría al respecto, pues, como advertimos, la bibliografía encontrada y seleccionada nos parece insuficiente para alcanzar la categorización selectiva (Strauss y Corbin, 2002), intentaremos mostrar algunos puntos de convergencia entre las investigaciones analizadas respecto de las principales barreras que limitan la participación en las escuelas.

En primer lugar, aparecen aquellos obstáculos propios del quehacer docente, particularmente el escaso tiempo con que cuentan los maestros para el desarrollo de proyectos e instancias que propicien adecuada y verdaderamente la participación, la ciudadanía y la democracia (Flanagan et al., 2010; Muñoz, 20I I; Martínez et al., 2010) y la presión por conseguir buenos resultados en las evacuaciones estandarizadas (Muñoz, 20I I; Redón, 20II), en tanto la calidad de la educación se ha limitado a la medición de contenidos académicos: "la formación ciudadana no posee una posición clara en el currículo y tiene que ser sacrificada frente a otros contenidos de carácter prioritario (e.g., ciencias y matemáticas), y esta situación también se refleja en la escasa medición de estas competencias en otros instrumentos (SIMCE), recursos asignados, indicadores de desempeño y estatus para el establecimiento educacional" (Martínez et al., 20I0, p. I I2). En efecto, la extensión de los contenidos académicos, la sobrecarga de los docentes en determinados contextos escolares y la exigencia de cumplir con evaluaciones que no se interesan por las aptitudes ciudadanas, terminan por mermar el desarrollo de la participación, de los procesos democráticos en las escuelas, desincentivando a los estudiantes, lo que según algunos actores sería propiciado por parte de las políticas públicas: "se destaca la opinión de los profesores, quienes indican que este desinterés por parte de los jóvenes se fomenta por el gobierno" (Flanagan et al., 2010, p. I3I).

Tanto el escaso tiempo como la presión por obtener resultados en evaluaciones estandarizadas serían consecuencia, como decíamos, de las políticas públicas, de una macropolítica que no busca que los estudiantes participen activamente, a pesar de instancias como el consejo escolar: "los significados construidos desde la macropolítica 
tienen una incidencia mayor en la cultura del Consejo Escolar y del establecimiento" (Muñoz, 20II, p. 47), y en este sentido, sería fundamental el apoyo que pueda prestar el MINEDUC (Martínez et al., 2010) para potenciar, en la práctica, espacios de participación y formación ciudadana.

En segundo lugar, las mismas prácticas pedagógicas de los maestros se convierten en obstáculos para fomentar la participación y desarrollar la ciudadanía, puesto que esas políticas se traducen en una labor pedagógica reproductiva y transmisiva, que apenas permite que los estudiantes participen de verdad, a la vez que instaura una mirada adultocéntrica y autoritaria (Flanagan et al., 2010; Martínez et al., 20I0; Muñoz, 20I I; Redón, 20I I). Este punto se manifiesta, por ejemplo, en la falta de sentido que tienen para los estudiantes las normas y reglas impuestas por la escuela dentro y fuera del aula: "ante la pregunta a los estudiantes respecto de si el establecimiento presenta contextos y situaciones en las cuales el estudiante puede elegir, ellos responden que no, y que solo es posible hacerlo en el recreo", pues "los niños y niñas al estar fuera de la supervisión y autoritarismo del docente, practican valores ciudadanos correspondientes al ámbito del espacio común, como el respecto, la tolerancia, la capacidad organizativa, la solidaridad” (Redón, 20II, p. 463).

Es así como los docentes adquieren una relevancia fundamental en este campo (Prieto, 2005; Cárcamo, 2008; Muñoz, 20II; Redón, 20II), ya que en buena medida depende de ellos permitir o limitar la participación de los estudiantes y fomentar el ejercicio de la ciudadanía:

la participación de los estudiantes en el aula está condicionada por una serie de factores. Por una parte, dependerá de las significaciones de los profesores acerca de ésta, las que, a su vez, estarán influenciadas por las racionalidades que informan sus prácticas docentes. Por otra, dependerá de la naturaleza de las oportunidades que propicia el profesor para que los estudiantes se puedan o no involucrar activamente en sus procesos formativos (Prieto, 2005, pp. 28-29)

En general, hay una coincidencia en que los profesores más bien restringen y dificultan estas actividades (Prieto, 2005; Martínez et al., 
20I0; Muñoz, 20I I; Redón, 20I I), algo que podría estar vinculado con una formación universitaria entregada a ciertos maestros alejada de estos intereses, tal como lo afirma Díaz Barrera (2010) al referirse a las escuelas inclusivas.

Finalmente, las investigaciones también tienen como punto en común vincular estas evidencias con un sistema político-económico neoliberal que no tendría mayor interés en la formación ciudadana (y en la construcción de comunidades), antes bien, profundizaría la individualidad, la competencia, la segregación y la exclusión, descuidando la formación ciudadana de la formación escolar, al establecer y legitimar prácticas guiadas por una racionalidad instrumental, opuesta a la racionalidad dialógica necesaria para desarrollar la participación y la formación ciudadana (Prieto, 2005; Díaz Barrera, 2010; Flanagan et al., 2010; Muñoz, 20I I; Redón, 20II). Esto último es explicado claramente en una sistematización de estudios previos que busca establecer las características del "modelo dialógico de la pedagogía”, realizada por Ferrada y Flecha (2008, p. 45), afirmado que la educación:

se comprende como un medio para promover interacciones dirigidas a transformar las propias construcciones intersubjetivas de quienes participan en el acto educativo al interior de una comunidad con predominio de la racionalidad comunicativa. Esto fundamenta que quienes participan adquieren protagonismo para intervenir en cada una de las decisiones educativas, mediante las interacciones que mantienen entre sí para la construcción de nuevos significados cuando deciden emprender proyectos conjuntos dirigidos a transformar la escuela.

En suma, la formación ciudadana no lograría su objetivo, ya que la participación del estudiantado está restringida por diversas razones: los niños y jóvenes serían vistos como objetos y no como sujetos que pueden aportar con sus significados a la construcción del espacio común y predominaría un proceso formativo academicista y autoritario, dirigido por políticas educativas que tienden más bien a dificultar el desarrollo de la ciudadanía. Por tanto, las escuelas, más que propiciar los procesos de participación y de construcción 
de ciudadanía, transmitirían y legitimarían estereotipos y valores tributarios del neoliberalismo y de una racionalidad instrumental que obstaculiza tales procesos.

\section{La familia en la escuela}

La segunda tendencia que logramos identificar en las investigaciones chilenas sobre el vínculo escuela-comunidad, pone el foco en la inclusión/exclusión de las familias en esa supuesta comunidad escolar. A pesar de haber encontrado escasos trabajos que cumplieran con los requisitos definidos para este artículo sobre la relación escuela-familia-comunidad, sin duda que se trata de un fenómeno de suma importancia, pues parte de los trabajos existentes en esta línea (Brunner y Elaqcua, 2004; Bellei et al, 2007, en Ansaldo San Martín, 20I5) vinculan la importancia de la familia y la participación de esta en la escuela con la mejora en los aprendizajes de los estudiantes. En este sentido, los resultados entregados por las investigaciones aquí seleccionadas vienen a respaldar esto. Pero también se señala la importancia atribuida a la participación de las familias como integrantes de la comunidad escolar para la formación ciudadana del estudiantado, lo que contrasta con la percepción de escasos espacios de participación existentes.

Las investigaciones de Flanagan et al. (2010) y Martínez et al. (2010), que ahondan en la comunidad escolar como espacio para la formación ciudadana, dan cuenta de la importancia que jugaría la familia en tanto integrante de una comunidad. Para Martínez et al. (2010) los programas que buscan desarrollar la ciudadanía en el estudiantado deben trabajar en conjunto con las familias de los estudiantes, sobre todo en contextos de vulnerabilidad. En este sentido, tanto estudiantes como docentes reconocen al hogar como un espacio de formación ciudadana, pues en la educación entregada por los padres se encontrarían las bases del desarrollo personal (Flanagan et al., 2010).

En el estudio de Rivera y Milicic (2006) los padres valoran su propia participación en la escuela para que esta devenga comunidad, pues los estudiantes desarrollarían habilidades sociales y flexibilidad frente a la diversidad presente en los centros educativos. Estos padres 
conciben la participación como una instancia de organización en la que se trabaja por el bien común, contagian a sus hijos con las ganas de participar, pues se ven a sí mismos como modelos, brindando a sus hijos una oportunidad de integración y desarrollo, tanto para ellos como para la comunidad.

Tal como la familia es percibida como un actor importante para el desarrollo del ejercicio democrático en los estudiantes, en segundo lugar es considerada un factor clave para la mejora en la calidad de la educación y el logro de aprendizajes (Rivera y Milicic, 2006; Gubbins, 2012), estrechando así el vínculo entre escuela y comunidad desde la perspectiva del cumplimiento efectivo del aprendizaje, tal como lo han hecho estudios previos (Brunner y Elaqcua, 2004; Bellei et al., 2007). En este sentido, Ferrada y Flecha (2008) hacen una sistematización de estudios sobre las comunidades de aprendizaje -en el que emergería un modelo dialógico de la pedagogía-, cuya principal característica es que toda la comunidad se compromete activamente para que todos los estudiantes logren aprendizajes de calidad. Por otro lado, Rivera y Milicic (2006) toman como antecedentes de su investigación algunos estudios que reconocen el vínculo entre implicación de la familia en el proceso de aprendizaje y el éxito escolar de los estudiantes. Desde esta perspectiva, el valor de la participación de los padres se asocia con la mejora en los resultados de los estudiantes, principalmente en contextos de vulnerabilidad. Sin embargo, a pesar del papel que pueden desempeñar las familias y la positiva percepción que se tiene de su participación, tal parece que, en la práctica y a través de las políticas públicas, "se desconoce la necesidad que llegue al aula para enriquecer los aprendizajes” (Rivera y Milicic, 2006, p. I20). Esta dificultad en las relaciones entre escuela y familia, en vistas del aprendizaje, ya había sido observada "en el estudio "¿Quién dijo que no se puede? Escuelas efectivas en sectores de pobreza” (Bellei et al, en Ansaldo San Martín, 2015).

La importancia atribuida tanto por docentes como por los propios padres y apoderados y la evidencia presentada en las investigaciones citadas que afirman la relación entre implicación de la familia y mejora en los resultados escolares, parece no ser suficiente argumento para que los padres y apoderados se integren activamente a las escuelas, pues los 
estudios aquí revisados muestran que la participación de la familia, al igual que en el caso de los estudiantes en el contexto de la formación ciudadana, es limitada, concordando con los resultados de la encuesta realizada por el CIDE el año 20I2, la que muestra que "casi un 60\% de los apoderados encuestados indica una participación baja o media en actividades o reuniones con profesores, un 75,3 participación baja o media en actividades extra-programáticas y un 50\% indica un nivel de participación baja o media para conversar temas disciplinarios con el profesor" (Ansaldo San Martín, 2015, p. 69).

Existirían al menos dos niveles de participación restringidos a actividades más bien simbólicas: "desde una lógica de rendición de cuentas externa o impuesta desde afuera del establecimiento educacional $[\ldots]$ y otra de carácter interno o carácter autorregulativo [...] Ambos procesos de participación se asocian a una lógica de control de eficacia de las escuelas" (Gubbins, 2012, p. 65). En los dos niveles se trataría, por tanto, de controlar la gestión administrativa: con la elección de la escuela, en el primer caso, y con la mejora en procesos internos y estándares de las escuelas, relegando a los padres a un papel secundario en el proceso educativo, pese a que en algunos casos se les exige cumplir con determinadas tareas y obligaciones y se les reprocha su ausencia (Rivera y Milicic, 2006); lo que parece contradictorio, privándolos de una participación auténtica que les permita tomar decisiones como actores legitimados en la escuela.

De este modo, nos enfrentamos a un discurso que enarbola la importancia de la comunidad, pero que no se estaría desplegando en la práctica: "lo que se tiene, entonces, es que no obstante el discurso promocional de alianza y democratización de la gestión escolar ello no ha permeado lo suficiente la toma de decisiones a nivel pedagógico y administrativo de las escuelas"(Gubbins, 2012, p. 65), a pesar de que ha existido intentos en la historia de quebrar este paradigma, tratando de fomentar una participación deliberativa. Lo anterior se manifiesta, por ejemplo, en que las familias no saben cómo participar, la comunicación con los apoderados es unidireccional y los escasos encuentros se limitan al traspaso de información principalmente académica y conductual por parte de los profesores a los padres (Rivera y Milicic, 2006), porque una participación de carácter 
deliberativo por parte de las familias es considerado problemático (Gubbins, 2012).

Esta limitada participación estaría emparentada con las expectativas y roles que familias y profesores se asignan mutuamente, lo que produciría la exclusión de los padres y apoderados de su integración al mundo escolar, pues los profesores, en general, acusan un abandono de los estudiantes:

Para los profesores (la educación) comienza en el hogar cuando los padres se hacen cargo de transmitir valores y normas a los hijos y procuran que respondan en la escuela. En cambio, los padres conciben este trabajo conjunto familia-escuela en planos separados, de modo que mientras ellos trabajan para cubrir necesidades y mantener a sus hijo. (Rivera y Milicic, 2006, p. I24).

Es decir, los docentes perciben que los apoderados no asumen su responsabilidad en el desempeño de sus hijos, y, por su parte, los apoderados sienten que los profesores les traspasan toda la responsabilidad. Un hallazgo similar al de Ansaldo San Martín (2015, p. 75):

Los actores profesionales de los establecimientos (directivos y docentes) en general señalan que es labor de la escuela inculcar ciertos valores, pero que es complejo porque los estudiantes vienen con "otros" desde su entorno familiar. En general hay poca claridad de qué valores quiere o debe transmitir el establecimiento, tanto para adultos como para estudiantes.

Por otro lado, al igual que en las investigaciones sobre ciudadanía, a los profesores se les otorga un papel central para el desarrollo de la participación, pues dependería en buena medida de ellos invitar a las familias a participar en la escuela o excluirlas: un buen profesor, permitiría la integración de los apoderados. Aquellos padres que se consideran participativos declaran tener una relación directa con los maestros, mientras que los que no participan sienten un trato prepotente de los docentes, excluyéndolos aún más. Sin embargo, al parecer, demasiado acercamiento por de los padres es considerado una intromisión por parte del profesorado (Rivera y Milicic, 
2006). Así, se podría afirmar que de las dos lógicas por las que está atravesada la participación de las que nos habla Gubbins (2012) prima una escuela cerrada, administrada por expertos, donde los padres controlan la eficacia, ya que para esta autora las políticas que promueven la participación no se han logrado traducir en prácticas más democráticas.

Las razones a las que se les atribuye esta de la falta de participación ya han sido mencionadas en parte: la vulnerabilidad social, la predominancia de una "lógica de los expertos", las aprehensiones de docentes y directivos que miran con recelo las prácticas deliberativas, a las que se suma el escaso tiempo disponible por parte de familias y docentes, además de la búsqueda individual del bienestar económico y las altas exigencias laborales que haría incompatible para los padres su trabajo con la escuela (Rivera y Milicic, 2006). Todo este complejo panorama representaría "una de las grandes contradicciones del sistema educacional chileno actual: se fomenta una participación más activa de las familias, pero desde una lógica de mercado" (Gubbins, 20I2, p. 46), llevando a las familias a vincularse con la escuela desde una postura más cercana a la del cliente-consumidor.

\section{A modo de conclusión}

¿Nuestras escuelas son comunidades educativas? Como decíamos al inicio, parece ser un lugar común referirse a la escuela como comunidad educativa o como comunidad escolar; sin embargo, a la luz de los resultados de este artículo que se ha propuesto revisar la bibliografía nacional existente, tal parece que las escuelas no son comunidades, al menos no en el sentido que hemos definido el concepto, pues las investigaciones aquí revisadas señalan que no presentan las características propias para ser consideradas tales. Tal conclusión es coherente con el resultado del estudio de Ansaldo San Martín (2015, p. 79) quien propone que: "confirmamos que existe entre los entrevistados una visión de comunidad escolar, entendida desde quiénes son sus miembros y no desde el tipo de relaciones y vinculación que hay entre ellos, lo que no impide que en el discurso se aprecie su importancia y relevancia para el desarrollo de la escuela $[\ldots]$. Se observó que las comunidades escolares son efímeras y de 
poco peso para las personas. Cuando se habla de comunidad en realidad es una etiqueta para adjudicar ciertas características deseables, más presentes en los discursos que en prácticas o hechos concretos. Lo que le da gravidez y sustancia a las comunidades, es decir el elemento afectivo, solidario y ético, que permite generar lazos con una carga de compromiso, no existe en las comunidades escolares revisadas".

Las concepciones y percepciones de una participación limitada, el escaso poder de decisión sobre asuntos importantes por parte de los estudiantes y las barreras para el diálogo y la incorporación de las familias en la construcción simbólica de la escuela, entre otros elementos, dan cuenta de ello. Esta observación es manifestada por Prieto (2005, p. 28), quien afirma que "no se ha visualizado aún la necesidad de transformarla [la escuela] en una comunidad en la que todos tengan derecho a participar”. Esta constatación, lejos de ser inocua, es signo de la importancia de investigar directamente el vínculo escuela y comunidad, en tanto representaría tanto una oportunidad para mejorar los aprendizajes en general como para potenciar el desarrollo de habilidades y valores democráticos en particular.

También nos preguntamos qué nos dice la investigación educativa en Chile sobre el vínculo escuela-comunidad y lo primero que se puede constatar es que el foco de los estudios no se ha posado lo suficiente sobre esta dimensión, pues la publicación de investigaciones y trabajos en revistas indexadas es aún insuficiente como para hacer generalizaciones o desarrollar políticas públicas que enfrenten este desafío. Por otra parte, se vislumbra cierta constante en términos metodológicos, en tanto la mayoría de las investigaciones son de corte cualitativo, que buscan comprender los significados de los actores, principalmente de los estudiantes. Quizá sea necesario desarrollar trabajos etnográficos para ir más allá de las representaciones de los actores de la institución escolar y analizar qué ocurre al interior de las escuelas, complementar con estudios cuantitativos que puedan abarcar un mayor número de sujetos para tener resultados generalizables y ampliar el foco de las investigaciones hacia los docentes y equipos directivos, con el fin de tener un panorama más amplio del tema, identificar causas y esbozar propuestas para que las 
escuelas se transformen en comunidades verdaderas, enriqueciendo el conocimiento producido hasta ahora.

No obstante lo anterior, en la presente revisión bibliográfica se pueden distinguir al menos dos tendencias temáticas: "ciudadanía y participación” y "familia y aprendizaje”. En ambas se destaca, tanto por parte de los autores como por los sujetos analizados (estudiantes, profesores y familias), que existe una positiva valoración de la participación de los integrantes de las escuelas, con objetivos como la formación ciudadana, el desarrollo y la práctica democrática y el logro de aprendizajes de calidad. Mas, como decíamos, en la cotidianidad de la vida escolar esa participación sería restringida y simbólica, volviéndose contradictoria, o al menos insuficiente, con el discurso educativo del Estado que destaca la importancia del aprendizaje democrático, lo que, a nuestro juicio, vuelve necesario seguir investigando, presentando y evaluando proyectos para abrir nuevos espacios de participación y mejorar los ya existentes.

Por último, como indicio de una dimensión más profunda y compleja, cabe destacar el papel preponderante que se le atribuye a los docentes, pues estos, en el papel de ejecutores directos de las políticas públicas y representantes del autoritarismo y el adultocentrismo de las escuelas, son percibidos como uno de los responsables por la escasa participación, ya que al interior de las aulas ellos reproducen prácticas y transmiten información, limitando la interacción de los estudiantes. Y si son, al menos en parte, responsables, también tendrían la posibilidad de generar y potenciar espacios de participación y cooperar de este modo con la democratización de la escuela. Los docentes tendrían en sus manos la posibilidad de permitir o negar la participación a través de sus prácticas pedagógicas, pasando por sus concepciones sobre la participación y, por último, por el tipo de racionalidad que guía su actuar, el que según algunos autores debiese ser una racionalidad comunicativa. Desde esta perspectiva, es relevante reflexionar en torno a la formación docente, tanto inicial como continua -sobre todo en el actual contexto en el que la pedagogía se ha vuelto objeto de análisis y discusión, por ejemplo, en el proyecto de una nueva carrera docente, los estímulos para estudiar pedagogía y el 
cuestionamiento a la calidad de la formación y del ejercicio del profesorado- si se quiere lograr que las escuelas sean efectivamente espacios democráticos, participativos, de educación ciudadana, en otras palabras, que la escuela sea una comunidad.

\section{Referencias bibliográficas}

Anderson, G. (2002). Hacia una participación auténtica. Deconstrucción del discurso de las reformas participativas en educación. En M. Naradowsky. Nuevas tendencias de las políticas educativas. Estado, mercado y escuela. Buenos Aires: Editorial Granica.

Ansaldo San Martín, S. (2015). Comunidad educativa escolar en la Modernidad Líquida. Comunicación y Medios, 32.

Bauman, Z. (2012). Modernidad líquida. Buenos Aires: Fondo de Cultura Económica.

Brunnner, J. J. y Elacqua G. (2004). Factores que inciden en una educación efectiva. Evidencia internacional. Revista Virtual La Educación, XVLIIIXLIX(I39-I40), I-II.

Cárcamo, H. (2008). Importancia atribuida al desarrollo de la ciudadanía en la formación inicial docente. Estudios Pedagógicos, 34(2), 29-43.

Díaz, N. (2010). Escuela inclusiva: revolución educativa en la construcción democrática de la sociedad en Chile. Revista Intercontinental de Psicología y Educación, I2(2), 47-63.

Ferrada, D. y Flecha, R. (2008). El modelo dialógico de la pedagogía: un aporte desde las experiencias de las comunidades de aprendizaje. Estudios Pedagógicos, 34(I), 4I-6I.

Flanagan, A. et al. (2010). Tensiones y distensiones en torno a la ciudadanía y formación ciudadana: comparación de los significados de profesores y estudiantes secundarios en la región de Valparaíso. Última Década, 33, II5-I37.

Gubbins, V. (2012). Familia y escuela: tensiones, reflexiones y propuestas. Docencia, 46, 64-73.

Krichesky, M. (2006). Escuela y comunidad, desafios para la inclusión educativa. Buenos Aires: Ministerio de educación, Ciencia y Tecnología de la Nación.

Martínez, M. L. et al. (2010). Los jóvenes ciudadanos: reflexiones para una política de formación ciudadana juvenil. Última Década, 32, I05-I I8.

Ministerio de Educación de Chile. (2009). Establece la Ley general de Educación. 
Recuperado el 4 de octubre de 2013 de http://www.leychile.cl/ Navegar?idNorma=I006043.

Muñoz, G. (20II). Representación simbólica de los consejos escolares como estrategia para democratizar la cultura escolar: una lectura interpretativa desde la voz de los sujetos sociales. Estudios Pedagógicos, 37, I, 35-52.

Neirotti, N. y Poggi, M. (2004). Alianzas e innovaciones en proyectos de desarrollo educativo local. Buenos Aires: IIPE-UNESCO.

Prieto, M. (2005). La participación de los estudiantes: ¿un camino hacia su emancipación? Theoria, I4(I), 27-36.

Redón, S. (2010). La escuela como espacio de ciudadanía. Estudios Pedagógicos, $36(2), 213-239$.

Redón, S. (20II). Escuela e identidad: un desafío para la cohesión social. Revista de la Universidad Bolivariana, IO(30), 447-476.

Rivera, M. y Milicic, N. (2006). Alianza familia-escuela: percepciones, creencias, expectativas y aspiraciones de padres y profesores de enseñanza general básica. Psykhe, I, I I9-I35.

Roussos, A. (20II). Preparación de una revisión bibliográfica para su publicación. Cuando un solo artículo nos habla de muchos trabajos. Reportes de Investigación, I, I-7.

Touraine, A. (2012). Crítica de la modernidad. México: Fondo de Cultura Económica. 